\title{
Thin HfSiN Films prepared by Magnetron Sputtering
}

\author{
ZaiyuZhang $^{1,2,3}$,Yilong Liang ${ }^{1,2^{*}}$, Xianbang Jiang $^{3}$
}

1 .College of Materials and Metallurgy, Guizhou University, Guiyang 550025, China;

2. Key Laboratory for material structure and strength of Guizhou province, Guizhou Guiyang 550025 ,

3. School of Electronic and Inform Engineering, Anshun University, 516000, Anshun, Guizhou. a E-mail:zaiyuzhang@126.com, b E-mail: liangyilong@126.com

Keywords: HfSiN thin films; magnetic sputtering; HfSiN/Cu/HfSiN/SiO2/Si

Abstract. HfSiN thin films were prepared by the solid solution of HfN and SiN precursor films through magnetron sputtering. The obtained films were characterized using X-ray diffraction (XRD) and scanning electron microscopy (SEM). X-ray diffraction(XRD) measurements show that the films have amorphous structure in the as-deposited state. Scanning electronic microscopy (SEM) images show that crystalline grain size of the films increases with the annealing temperature. The results show that the resistivity and the components of the $\mathrm{HfSiN} / \mathrm{Cu} / \mathrm{HfSiN} / \mathrm{SiO} 2 / \mathrm{Si}$ film do not have obvious change after being annealing at $550^{\circ} \mathrm{C}$ in oxygen, and the HfSiN film can provide good barrier performance for copper wire.

\section{Introduction}

Copper has been extensively studied as an interconnect material for deep sub-micron circuits due to its superior electrical resistivity and resistance to electromigration. A suitable diffusion barrier is crucial to suppress the diffusion and reaction taking place between copper and silicon at low temperature for realizing thermally stable copper wiring. Refractory metal binary and ternary nitrides are widely recognized as a class of materials which can be used as diffusion barriers in metal-semiconductor contacts[1]. Among those refractory metal nitrides, zirconium-base and tantalum-base nitride thin films, such as $\mathrm{ZrSiN}$ and Ta-Si-N, have been extensively studied as diffusion barriers for copper wiring[2-5]. Although $\mathrm{ZrSiN}$ and $\mathrm{TaSiN}$ barriers exhibit good performance as diffusion barriers, high resistivity limits their microelectronic applications. HfSiN is a new type of diffusion barrier. This films have high temperature of the crystallization and low charge trap density[6-8]. Herein, different deposition temperature and ratio of N2/Ar were investigated on the effect of the HfSiN film resistivity, and the $\mathrm{HfSiN} / \mathrm{Cu} / \mathrm{HfSiN} / \mathrm{SiO} 2 / \mathrm{Si}$ structures were prepared and annealed at temperatures varying from 500 to $600^{\circ} \mathrm{C}$.

\section{Experiment}

The specimens were prepared in JPG500A Multi-Function UHV Magneteon Sputtering System. In order to remove organic contaminants and native oxide, substrates of Si were successively cleaned in an ultrasonic bath with diluted HF solution and then rinsed in deionized water before the activation process. The deposition system was executed under a vacuum condition lower than $6 \times 10-4 \mathrm{~Pa}$ prior to deposition. During the deposition process, the pressure in vacuum chamber was $4 \mathrm{~Pa}$ and the sputtering power is $40 \mathrm{~W}$. The substrate bias voltage was varied from 40 to $160 \mathrm{~V}$ without substrate heating.

Main target material uses Hf target and silicon target. The purity of target material is $99.99 \%$.HfN and $\mathrm{SiN}$ films composed $\mathrm{HfSiN}$.In order to achieve $45 \mathrm{~nm} \mathrm{HfSiN} / \mathrm{Cu} / \mathrm{HfSiN} / \mathrm{SiO} 2 / \mathrm{Si}$ structures, $5 \mathrm{~nm}$ HfSiN films were deposited on the Si substrates by magnetron sputtering, $35 \mathrm{~nm}$ Copper films covered on the HfSiN films, then $5 \mathrm{~nm}$ HfSiN films covered on $\mathrm{Cu}$ films. Three HfSiN/Cu/HfSiN/SiO2/Si specimens were annealed at $500{ }^{\circ} \mathrm{C}, 550^{\circ} \mathrm{C}$ and $600^{\circ} \mathrm{C}$ for an hour in oxygen ambient, respectively. XRD was used to determine the mass density of the gas-deposited and annealed samples, and it was 
operated at $40 \mathrm{kv}$ and $40 \mathrm{~mA}$, with $\mathrm{Cu}-\mathrm{K} \alpha$ radiation The surface of the barrier samples was characterized by a scanning electron microscopy (SEM), and it was operated at $30 \mathrm{kv}$.

\section{Results and Discussion}

\section{N2/Ar ratio on HfSiN film resistivity}

As can be seen from Figure 1, when the proportion of nitrogen increases, HfSiN resistivity increases. When the proportion of nitrogen is lower, more Hf atoms deposited on the Si substrate, forming the thin film of $\mathrm{Hf}$ content is high, a relatively low resistivity of the film; with $\mathrm{N} 2$ / (N2 + Ar) increase in the proportion, Hf, and N2 to increase the intensity of the reaction on the substrate is formed of a high resistance value compound film.

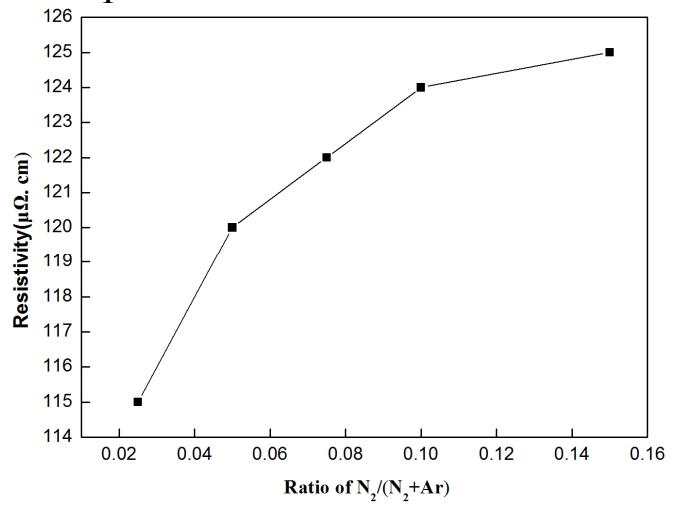

Fig. 1 Electrical resistivity of HfSiN film as a function of nitrogen proportion

\section{Effect of temperature on HfSiN film}

Figure 2 is deposited at different temperatures HfSiN film resistivity change. As can be seen from the Figure 2, the resistivity of the film decreases with the increase of the operating temperature. When the temperature was raised to $400{ }^{\circ} \mathrm{C}$, resistance of the resulting film was $100 \mu \Omega \cdot \mathrm{cm}$. When the temperature is low, the critical nucleation free energy is small, and the number of core grains formed in the formation of many small, poor crystallinity, greater resistivity.

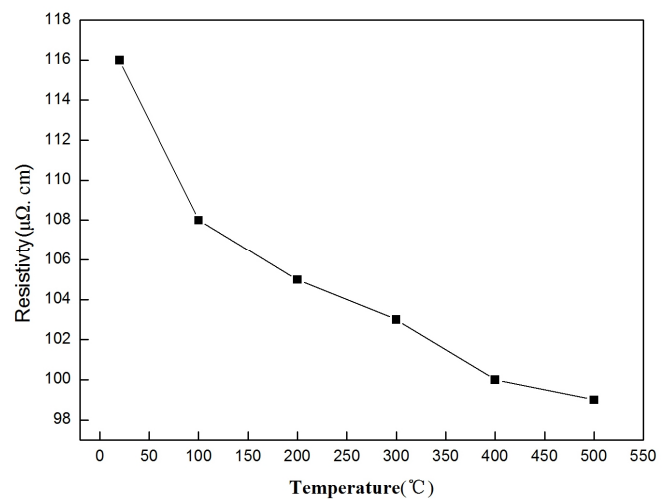

Fig.2 Electrical resistivity of HfSiN film as different temperature

\section{XRD analysis of the multi-layer film before and after annealing}

Figure 3(baselayer) is a diffraction pattern of multilayer film ( $\mathrm{HfSiN} / \mathrm{Cu} / \mathrm{HfSiN} / \mathrm{SiO} 2 / \mathrm{Si}$ ) before annealing by XRD. There is only the elemental Hf(111) peaks, which indicates that amorphous Hf has been deposited on the silicon substrate. Figure 3(interlayer) shows that $\mathrm{CuO}(111) \mathrm{CuO}(-111)$ and $\mathrm{HfO} 2$ appear in the multilayer film after being annealed at $550{ }^{\circ} \mathrm{C}$. . This peaks explain $\mathrm{Cu}$ and $\mathrm{Hf}$ start oxidation. When the sample annealed at $600{ }^{\circ} \mathrm{C}$ in the Figure 3(toplayer), in addition to $\mathrm{CuO}(111)$ and $\mathrm{CuO}(-111)$ diffraction peak, the sample also appeared $\mathrm{Si}(111)$ diffraction peak. After being annealed at $550{ }^{\circ} \mathrm{C}$, copper diffusion occurs, HfSiN film of copper diffusion barrier properties of the gradual failure; After annealing at $600{ }^{\circ} \mathrm{C}$ lot of copper diffusion occurs through HfSiN layer, forming a large amount of $\mathrm{CuO}$, this time $\mathrm{HfSiN}$ copper diffusion barrier properties of completely failure. 


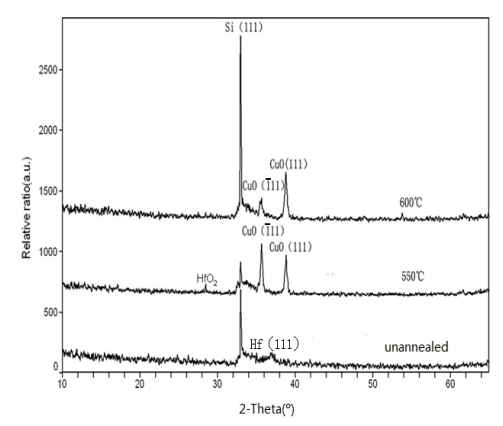

Fig.3 XRD of $\mathrm{HfSiN} / \mathrm{Cu} / \mathrm{HfSiN} / \mathrm{SiO} 2 / \mathrm{Si}$ film before and after annealing

\section{SEM images of multilayer films before and after annealing}

As can be seen from Figure 4, in the multilayer film without annealing $\mathrm{HfSiN} / \mathrm{Cu} / \mathrm{HfSiN} / \mathrm{SiO} 2 / \mathrm{Si}$ smooth surface without generating large particles to exist as amorphous. Under $550{ }^{\circ} \mathrm{C}$ high temperature annealing to form a small number of large particles. At $600{ }^{\circ} \mathrm{C}$ generate a large number of large particles, then the following $\mathrm{HfSiN}$ thin $\mathrm{Cu}$ grain boundary diffusion through the large surface of the $\mathrm{O}$ to form a copper oxide, leading to a sharp rise in resistivity, resulting in failure of the barrier layer.
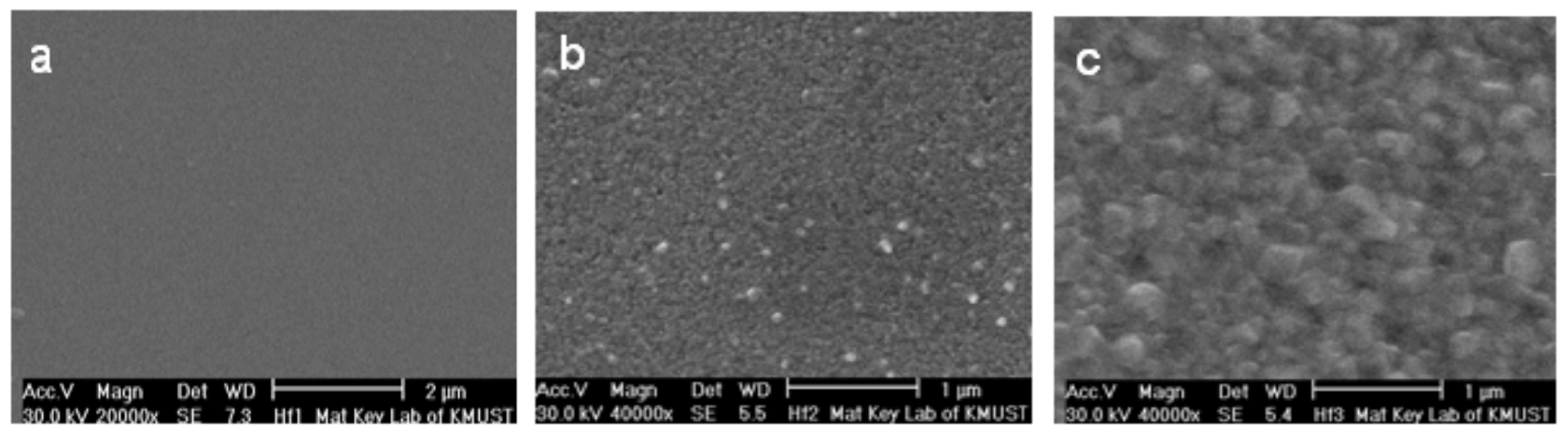

Fig.4 SEM of HfSiN/Cu/HfSiN/SiO$/ 2 / S i$ film before and after annealing

\section{Conclusions}

Under different experimental conditions HfSiN parameters and $\mathrm{HfSiN} / \mathrm{Cu} / \mathrm{HfSiN} / \mathrm{SiO} 2 / \mathrm{Si}$ multilayer films prepared by magnetron sputtering technique in this article. The effects of temperature, the ratio of nitrogen and argon prepared HfSiN film. Also investigated HfSiN films of $\mathrm{Cu}$ diffusion barrier properties under sub-45nm-class process conditions. The results show that the nitrogen content is $2.5 \%$ of nitrogen gas and argon gas mixture corresponding HfN film resistivity ratio is less than the resistivity of the other samplesUnder conditions of $550^{\circ} \mathrm{C}$ annealing in an oxygen atmosphere can be maintained good $\mathrm{HfSiN}$ film copper diffusion barrier properties. When the temperature exceeds $550{ }^{\circ} \mathrm{C}$, the resistivity increases sharply multilayers beyond the range, and the formation of $\mathrm{CuO}$ material, so that the film HfSiN ability to copper diffusion barrier failure.

\section{Acknowledgements}

This work was supported by the technology innovation find of the ministry science and technology in China(No.04C2615200858). This work was financially supported by Guizhou Branch of J word(LKA2013-15).

\section{Reference}

[1] Wang Yangyuan, Huang Ru, L iu Xiaoyan, et al. Physics, 2004; 33 : 407 - 413

[2] X.Qi, L.Yu, Jan Musschoot, et al. thin Solid Films 2009;517 : 4689-4693. 
[3] Dung-Ching Perng, Kuo-Chung Hsu, Shuo-Wen Tsai, et al. Microelectronic Engineering 2010;87:365-369.

[4] Y.Wang, Ch.H. Zhao,F. Cao,et al.Materials Letters2008;62:418-421.

[5] R.Hübner, M.Hecker, N.Matter, et al.Thin Solid Films2004; 468:183-192.

[6] Y. Wang, F. Cao, L.Shao, et al.Thin Solid Films2009; 517:5593-5596.

[7] Kashani-Shirazi, K.; Bachmann, A.; Boehm, G,etal.Materials Letters2009;311:1908-1911

[8]Gottwald, Matthias; Kan, Jimmy J.; Lee, Kangho,etal.Materials Letters2013;1(2):022102 